\title{
On Generalized Jordan Prederivations and Generalized Prederivations of Lie Superalgebras
}

\author{
Yao Ma and Liangyun Chen \\ School of Mathematics and Statistics, Northeast Normal University, Changchun 130024, China \\ Correspondence should be addressed to Liangyun Chen; chenly640@nenu.edu.cn
}

Received 10 May 2014; Revised 29 July 2014; Accepted 19 August 2014; Published 2 September 2014

Academic Editor: Andrei D. Mironov

Copyright ( 2014 Y. Ma and L. Chen. This is an open access article distributed under the Creative Commons Attribution License, which permits unrestricted use, distribution, and reproduction in any medium, provided the original work is properly cited.

\begin{abstract}
The concepts of (generalized) $(\theta, \varphi)$-prederivations and (generalized) Jordan $(\theta, \varphi)$-prederivations on a Lie superalgebra are introduced. It is proved that Jordan $(\theta, \varphi)$-prederivations (resp., generalized Jordan $(\theta, \varphi)$-prederivations) are $(\theta, \varphi)$-prederivations (resp., generalized $(\theta, \varphi)$-prederivations) on a Lie superalgebra under some conditions. In particular, Jordan $\theta$-prederivations are $\theta$-prederivations on a Lie superalgebra.
\end{abstract}

\section{Introduction}

Derivations and generalized derivations are interesting subjects both in mathematics and physics, and there has been a great deal of work concerning them. Leger and Luks investigated the structure of the generalized derivations of Lie algebras systematically (cf. [1]). Generalized derivations on rings were studied in [2, 3]. Generalized derivations also play a key role in Benoist's study of Levi factors in derivation algebras of nilpotent Lie algebras (cf. [4]). In 1969, Herstein showed that a Jordan derivation of a prime ring of characteristic not 2 must be a derivation (cf. [5]). Brešar together with Vukman generalized Herstein's result to Jordan $(\Theta, \varphi)$-derivations (cf. [6]). In [7], the authors proved that, in a 2-torsion free noncommutative prime ring $R$, a generalized Jordan $(\theta, \varphi)$-derivation is a generalized $(\theta, \varphi)$ derivation when $\theta$ is an automorphism of $R$. We gave some results on generalized derivations of Lie color algebras in [8]. Moreover, Jordan $\theta$-derivations and generalized Jordan derivations of Lie triple systems were studied in $[3,9,10]$.

Prederivations (or Lie triple derivations) of Lie algebras were first introduced by Müller to study bi-invariant semiRiemannian metrics on Lie groups. Let $G$ be a Lie group with a bi-invariant semi-Riemannian metric and $g$ its Lie algebra. Then the Lie algebra of the group of isometries of $G$ fixing the identity element is a subalgebra of the prederivation algebra of $g$ (cf. [11]). Bajo proved that a real or complex Lie algebra admitting a nonsingular prederivation is necessarily nilpotent (cf. [12]). Moens generated this result to Lie algebras over any field of characteristic zero in [13]. Burde showed that the existence of nonsingular prederivations is useful for the construction of the affine structure on Lie algebras (cf. [14]). In recent years, there has been an increasing interest in investigating prederivations (cf. $[2,15-20])$.

Lie superalgebras are the natural generalization of Lie algebras and have important applications both in mathematics and in physics. Lie superalgebras are also interesting from a purely mathematical point of view. So it is reasonable to extend the notion of prederivations to Lie superalgebras, which may do the same work in the structure of Lie superalgebras as the prederivations of Lie algebras did. In this paper, we introduce the concepts of (generalized) $(\theta, \varphi)$ prederivations and (generalized) Jordan $(\theta, \varphi)$-prederivations for a Lie superalgebra and obtain some results concerning Jordan $(\theta, \varphi)$-prederivations (resp., generalized Jordan $(\theta, \varphi)$ prederivations) and $(\theta, \varphi)$-prederivations (resp., generalized $(\theta, \varphi)$-prederivations) on a Lie superalgebra.

Throughout this paper, the base field $\mathbf{F}$ is assumed to be of characteristic not equal to 3 . We now recall some elementary definitions. 
Definition 1 (see $[12,19]$ ). A prederivation (Lie triple derivation) of a Lie algebra $L$ is a linear mapping $D: L \rightarrow L$ such that

$$
\begin{aligned}
D([[x, y], z])= & {[[D(x), y], z]+[[x, D(y)], z] } \\
& +[[x, y], D(z)], \quad \forall x, y, z \in L .
\end{aligned}
$$

Definition 2 (see [21]). A Jordan prederivation (Jordan triple derivation) of a Lie algebra $L$ is a linear mapping $D^{\prime}: L \rightarrow L$ such that

$$
\begin{aligned}
D^{\prime}([[x, y], x])= & {\left[\left[D^{\prime}(x), y\right], x\right]+\left[\left[x, D^{\prime}(y)\right], x\right] } \\
& +\left[[x, y], D^{\prime}(x)\right], \quad \forall x, y \in L .
\end{aligned}
$$

\section{Main Results}

Definition 3. Let $L$ be a Lie superalgebra and let $D, \theta, \varphi: L \rightarrow$ $L$ be homogeneous linear mappings, where $d(\theta)=d(\varphi)=0$. One denotes by $d(D)$ the $\mathbf{Z}_{\mathbf{2}}$-graded degree of a homogeneous linear mapping $D$ of $L$.

(1) $D$ is called a $(\theta, \varphi)_{1}$-prederivation if

$$
\begin{aligned}
D([[x, y], z])= & {[[D(x), \theta(y)], \varphi(z)] } \\
& +(-1)^{d(D) d(x)}[[\theta(x), D(y)], \varphi(z)] \\
& +(-1)^{d(D)(d(x)+d(y))} \\
& \times[[\theta(x), \varphi(y)], D(z)], \quad \forall x, y, z \in L .
\end{aligned}
$$

(2) $D$ is called a $(\theta, \varphi)_{2}$-prederivation if

$$
\begin{aligned}
D([[x, y], z])= & {[[D(x), \theta(y)], \theta(z)] } \\
& +(-1)^{d(D) d(x)}[[\theta(x), D(y)], \varphi(z)] \\
& +(-1)^{d(D)(d(x)+d(y))} \\
& \times[[\varphi(x), \varphi(y)], D(z)], \quad \forall x, y, z \in L .
\end{aligned}
$$

(3) $D$ is called a $(\theta, \varphi)_{3}$-prederivation if

$$
\begin{aligned}
D([[x, y], z])= & {[[D(x), \theta(y)], \theta(z)] } \\
& +(-1)^{d(D) d(x)}[[\varphi(x), D(y)], \theta(z)] \\
& +(-1)^{d(D)(d(x)+d(y))} \\
& \times[[\varphi(x), \varphi(y)], D(z)], \quad \forall x, y, z \in L .
\end{aligned}
$$

In particular, $\forall i=1,2,3$, a $(\theta, \varphi)_{i}$-prederivation $D$ is called a $\theta$-prederivation if $\theta=\varphi$. It is clear that a $(\theta, \varphi)_{i}$-prederivation is a prederivation when $\theta=\varphi=1_{T}$.

Definition 4. Let $L$ be a Lie superalgebra and let $D, \theta, \varphi: L \rightarrow$ $L$ be homogeneous linear mappings, where $d(\theta)=d(\varphi)=0$.
(1) $D$ is called a Jordan $(\theta, \varphi)_{1}$-prederivation if

$$
\begin{aligned}
D([[x, y], x])= & {[[D(x), \theta(y)], \varphi(x)] } \\
& +(-1)^{d(D) d(x)}[[\theta(x), D(y)], \varphi(x)] \\
& +(-1)^{d(D)(d(x)+d(y))} \\
& \times[[\theta(x), \varphi(y)], D(x)], \quad \forall x, y \in L .
\end{aligned}
$$

(2) $D$ is called a Jordan $(\theta, \varphi)_{2}$-prederivation if

$$
\begin{aligned}
D([[x, y], x])= & {[[D(x), \theta(y)], \theta(x)] } \\
& +(-1)^{d(D) d(x)}[[\theta(x), D(y)], \varphi(x)] \\
& +(-1)^{d(D)(d(x)+d(y))} \\
& \times[[\varphi(x), \varphi(y)], D(x)], \quad \forall x, y \in L .
\end{aligned}
$$

(3) $D$ is called a Jordan $(\theta, \varphi)_{3}$-prederivation if

$$
\begin{aligned}
D([[x, y], x])= & {[[D(x), \theta(y)], \theta(x)] } \\
& +(-1)^{d(D) d(x)}[[\varphi(x), D(y)], \theta(x)] \\
& +(-1)^{d(D)(d(x)+d(y))} \\
& \times[[\varphi(x), \varphi(y)], D(x)], \quad \forall x, y \in L .
\end{aligned}
$$

In particular, $\forall i=1,2,3$, a Jordan $(\theta, \varphi)_{i}$-prederivation $D$ is called a Jordan $\theta$-prederivation if $\theta=\varphi$. It is clear that a Jordan $(\theta, \varphi)_{i}$-prederivation is a Jordan prederivation when $\theta=\varphi=1_{T}$.

It is clear that if $D_{i}$ is a $(\theta, \varphi)_{i}$-prederivation of $L$, then $D_{i}$ is a Jordan $(\theta, \varphi)_{i}$-prederivation of $L$, where $i=1,2,3$.

In this section, $L$ is a Lie superalgebra and $\theta, \varphi$ are defined to be homogeneous linear mappings of $L$ satisfying $d(\theta)=$ $d(\varphi)=0$.

Theorem 5. $D$ is a $(\theta, \varphi)_{1}$-prederivation of $L$ if and only if $D$ is a Jordan $(\theta, \varphi)_{1}$-prederivation of $L$ such that

(i) $[[\theta(x), \varphi(y)], D(z)]=[[\varphi(x), \theta(y)], D(z)]$,

$$
\text { (ii) } \begin{aligned}
(-1)^{d(x) d(z)} A(x, y, z)+ & +(-1)^{d(x) d(y)} A(y, z, x) \quad+ \\
(-1)^{d(y) d(z)} A(z, x, y)=0, &
\end{aligned}
$$

where $x, y, z \in L$ and $A(x, y, z)=[[D(x), \theta(y)], \varphi(z)]+$ $(-1)^{d(D) d(x)}[[\theta(x), D(y)], \varphi(z)]+\quad(-1)^{d(D)(d(x)+d(y))}$ $[[\theta(x), \varphi(y)], D(z)]$. 
Proof. Assume that $D$ is a $(\theta, \varphi)_{1}$-prederivation of $L$. Clearly, $D$ is a Jordan $(\theta, \varphi)_{1}$-prederivation of $L$ and $D([[x, y], z])=$ $-(-1)^{d(x) d(y)} D([[y, x], z])$; note that

$$
\begin{aligned}
-(-1)^{d(x) d(y)} D([[y, x], z]) \\
=-(-1)^{d(x) d(y)}[[D(y), \theta(x)], \varphi(z)] \\
\quad-(-1)^{d(x) d(y)}(-1)^{d(D) d(y)} \\
\quad \times[[\theta(y), D(x)], \varphi(z)] \\
\quad-(-1)^{d(x) d(y)}(-1)^{d(D)(d(x)+d(y))} \\
\quad \times[[\theta(y), \varphi(x)], D(z)] \\
=[D(x), \theta(y), \varphi(z)]+(-1)^{d(D) d(x)} \\
\quad \times[[\theta(x), D(y)], \varphi(z)] \\
\quad+(-1)^{d(D)(d(x)+d(y))}[[\varphi(x), \theta(y)], D(z)] \\
D([[x, y], z]) \\
=[[D(x), \theta(y)], \varphi(z)] \\
\quad+(-1)^{d(D) d(x)}[[\theta(x), D(y)], \varphi(z)] \\
+(-1)^{d(D)(d(x)+d(y))} \\
\quad \times[[\theta(x), \varphi(y)], D(z)],
\end{aligned}
$$

then (i) follows. Since $D$ is a $(\theta, \varphi)_{1}$-prederivation of $L$, we have $D([[x, y], z])=A(x, y, z)$; hence

$$
\begin{aligned}
& (-1)^{d(x) d(z)} A(x, y, z)+(-1)^{d(x) d(y)} A(y, z, x) \\
& \quad+(-1)^{d(y) d(z)} A(z, x, y) \\
& =(-1)^{d(x) d(z)} D([[x, y], z]) \\
& \quad+(-1)^{d(x) d(y)} D([[y, z], x]) \\
& \quad+(-1)^{d(y) d(z)} D([[z, x], y]) \\
& =D\left((-1)^{d(x) d(z)}[[x, y], z]\right. \\
& \quad+(-1)^{d(x) d(y)}[[y, z], x] \\
& \left.\quad+(-1)^{d(y) d(z)}[[z, x], y]\right)=0 .
\end{aligned}
$$

Conversely, let $D$ be a Jordan $(\theta, \varphi)_{1}$-prederivation of $L$ for which (i) and (ii) hold. Then $D([[x, y], x])=A(x, y, x)$. It follows that

$$
\begin{aligned}
& D([[x+z, y], x+z]) \\
&= D([[x, y], x])+D([[x, y], z]) \\
&+D([[z, y], x])+D([[z, y], z])
\end{aligned}
$$

$$
\begin{aligned}
= & A(x, y, x)+A(z, y, z) \\
& +D([[x, y], z])+D([[z, y], x]), \\
D( & {[[x+z, y], x+z]) } \\
= & A(x+z, y, x+z) \\
= & A(x, y, x)+A(x, y, z) \\
& +A(z, y, x)+A(z, y, z) .
\end{aligned}
$$

Thus we obtain

$D([[x, y], z])+D([[z, y], x])=A(x, y, z)+A(z, y, x)$.

By (i),

$$
\begin{aligned}
A(y, & x, z) \\
= & {[[D(y), \theta(x)], \varphi(z)]+(-1)^{d(D) d(y)} } \\
& \times[[\theta(y), D(x)], \varphi(z)] \\
& +(-1)^{d(D)(d(x)+d(y))}[[\theta(y), \varphi(x)], D(z)] \\
= & -(-1)^{d(x) d(y)}[[D(x), \theta(y)], \varphi(z)] \\
& -(-1)^{d(x) d(y)}(-1)^{d(D) d(x)}[[\theta(x), D(y)], \varphi(z)] \\
& -(-1)^{d(x) d(y)}(-1)^{d(D)(d(x)+d(y))} \\
& \times[[\varphi(x), \theta(y)], D(z)] \\
= & -(-1)^{d(x) d(y)}[[D(x), \theta(y)], \varphi(z)] \\
& -(-1)^{d(x) d(y)}(-1)^{d(D) d(x)} \\
& \times[[\theta(x), D(y)], \varphi(z)] \\
& -(-1)^{d(x) d(y)}(-1)^{d(D)(d(x)+d(y))} \\
& \times[[\theta(x), \varphi(y)], D(z)] \\
= & -(-1)^{d(x) d(y)} A(x, y, z) .
\end{aligned}
$$

This implies that

$$
\begin{aligned}
D([[x, y], y]) & =-(-1)^{d(x) d(y)} D([[y, x], y]) \\
& =-(-1)^{d(x) d(y)} A(y, x, y) \\
& =A(x, y, y) .
\end{aligned}
$$

A similar argument proves

$$
D([[x, y], z])+D([[x, z], y])=A(x, y, z)+A(x, z, y) \text {. }
$$

By $(*) \times(-1)^{d(x) d(z)}+(* *) \times(-1)^{d(x) d(y)}$, we have

$$
\begin{aligned}
& (-1)^{d(x) d(z)}(D([[x, y], z])+D([[z, y], x])) \\
& \quad+(-1)^{d(x) d(y)}(D([[x, y], z])+D([[x, z], y]))
\end{aligned}
$$




$$
\begin{aligned}
= & (-1)^{d(x) d(z)}(A(x, y, z)+A(z, y, x)) \\
& +(-1)^{d(x) d(y)}(A(x, y, z)+A(x, z, y)) ;
\end{aligned}
$$

then

$$
\begin{aligned}
(-1)^{d(x) d(z)} D([[x, y], z]) \\
\quad+(-1)^{d(x) d(y)} D([[x, y], z]) \\
\quad-(-1)^{d(y) d(z)} D([[y, x], z]) \\
=(-1)^{d(x) d(z)}(A(x, y, z)+A(z, y, x)) \\
\quad+(-1)^{d(x) d(y)}(A(x, y, z)+A(x, z, y))
\end{aligned}
$$

that is,

$$
\begin{aligned}
&\left((-1)^{d(x) d(z)}+(-1)^{d(x) d(y)}+(-1)^{d(y)(d(x)+d(z))}\right) \\
& \quad \times D([[x, y], z]) \\
&=\left((-1)^{d(x) d(z)}+(-1)^{d(x) d(y)}+(-1)^{d(y)(d(x)+d(z))}\right) \\
& \quad \times A(x, y, z) \\
& \quad+(-1)^{d(x) d(z)} A(z, y, x) \\
& \quad+(-1)^{d(x) d(y)} A(x, z, y) \\
& \quad-(-1)^{d(y)(d(x)+d(z))} A(x, y, z) \\
&=\left((-1)^{d(x) d(z)}+(-1)^{d(x) d(y)}+(-1)^{d(y)(d(x)+d(z))}\right) \\
& \times A(x, y, z) \\
&+(-1)^{d(x) d(z)} A(z, y, x) \\
&+(-1)^{d(x) d(y)} A(x, z, y) \\
&+(-1)^{d(y) d(z)} A(y, x, z) \\
&=\left((-1)^{d(x) d(z)}+(-1)^{d(x) d(y)}+(-1)^{d(y)(d(x)+d(z))}\right) \\
& \times A(x, y, z),
\end{aligned}
$$

where the last equality uses (ii). Since $\operatorname{ch} \mathbf{F} \neq 3$, we have $(-1)^{d(x) d(z)}+(-1)^{d(x) d(y)}+(-1)^{d(y)(d(x)+d(z))} \neq 0$, and so $D([[x, y], z])=A(x, y, z)$; that is, $D$ is a $(\theta, \varphi)_{1}$-prederivation of $L$.

Corollary 6. $D$ is a $\theta$-prederivation of $L$ if and only if $D$ is a Jordan $\theta$-prederivation of $L$.

Proof. If $D$ is a Jordan $\theta$-prederivation of $L$, then (i) follows immediately. (ii) holds because

$$
\begin{aligned}
(-1)^{d(x) d(z)} A(x, y, z)+(-1)^{d(x) d(y)} A(y, z, x) \\
\quad+(-1)^{d(y) d(z)} A(z, x, y) \\
=(-1)^{d(x) d(z)}[[D(x), \theta(y)], \theta(z)]
\end{aligned}
$$

$$
\begin{aligned}
& +(-1)^{d(x) d(z)}(-1)^{d(D) d(x)}[[\theta(x), D(y)], \theta(z)] \\
& +(-1)^{d(x) d(z)}(-1)^{d(D)(d(x)+d(y))} \\
& \times[[\theta(x), \theta(y)], D(z)] \\
& +(-1)^{d(x) d(y)}[[D(y), \theta(z)], \theta(x)] \\
& +(-1)^{d(x) d(y)}(-1)^{d(D) d(y)} \\
& \times[[\theta(y), D(z)], \theta(x)] \\
& +(-1)^{d(x) d(y)}(-1)^{d(D)(d(y)+d(z))} \\
& \times[[\theta(y), \theta(z)], D(x)] \\
& +(-1)^{d(y) d(z)}[[D(z), \theta(x)], \theta(y)] \\
& +(-1)^{d(y) d(z)}(-1)^{d(D) d(z)}[[\theta(z), D(x)], \theta(y)] \\
& +(-1)^{d(y) d(z)}(-1)^{d(D)(d(x)+d(z))} \\
& \times[[\theta(z), \theta(x)], D(y)] \\
& =(-1)^{d(D) d(z)} \\
& \times\left((-1)^{d(z)(d(D)+d(x))}[[D(x), \theta(y)], \theta(z)]\right. \\
& +(-1)^{d(y)(d(D)+d(x))}[[\theta(y), \theta(z)], D(x)] \\
& \left.+(-1)^{d(y) d(z)}[[\theta(z), D(x)], \theta(y)]\right) \\
& +(-1)^{d(D) d(x)} \\
& \times\left((-1)^{d(x)(d(D)+d(y))}[[D(y), \theta(z)], \theta(x)]\right. \\
& +(-1)^{d(z)(d(D)+d(y))}[[\theta(z), \theta(x)], D(y)] \\
& \left.+(-1)^{d(x) d(z)}[[\theta(x), D(y)], \theta(z)]\right) \\
& +(-1)^{d(D) d(y)} \\
& \times\left((-1)^{d(y)(d(D)+d(z))}[[D(z), \theta(x), \theta(y)]]\right. \\
& +(-1)^{d(x)(d(D)+d(z))}[[\theta(x), \theta(y)], D(z)] \\
& \left.+(-1)^{d(x) d(y)}[[\theta(y), D(z)], \theta(x)]\right)=0 .
\end{aligned}
$$

Therefore, $D$ is a $\theta$-prederivation of $L$ by Theorem 5 .

Theorem 7. $D$ is a $(\theta, \varphi)_{2}$-prederivation of $L$ if and only if $D$ is a Jordan $(\theta, \varphi)_{2}$-prederivation of $L$ such that

(i) $[[D(x), \theta(y)],(\varphi-\theta)(z)]=(-1)^{d(D) d(x)}[[\theta(x), D(y)]$, $(\varphi-\theta)(z)]$

(ii) $(-1)^{d(x) d(z)} A^{\prime}(x, y, z)+(-1)^{d(x) d(y)} A^{\prime}(y, z, x)+$ $(-1)^{d(y) d(z)} A^{\prime}(z, x, y)=0$, 
where $x, y, z \in L$ and $A^{\prime}(x, y, z)=[[D(x), \theta(y)], \theta(z)]+$ $(-1)^{d(D) d(x)}[[\theta(x), D(y)], \varphi(z)] \quad+\quad(-1)^{d(D)(d(x)+d(y))}$ $[[\varphi(x), \varphi(y)], D(z)]$.

Proof. Let $D$ be a $(\theta, \varphi)_{2}$-prederivation of $L$. Use the fact that

$$
D([[x, y], z])=-(-1)^{d(x) d(y)} D([[y, x], z]),
$$

as well as the fact that

$$
\begin{aligned}
- & (-1)^{d(x) d(y)} D([[y, x], z]) \\
= & -(-1)^{d(x) d(y)}[[D(y), \theta(x)], \theta(z)] \\
& -(-1)^{d(x) d(y)}(-1)^{d(D) d(y)}[[\theta(y), D(x)], \varphi(z)] \\
& -(-1)^{d(x) d(y)}(-1)^{d(D)(d(x)+d(y))} \\
& \times[[\varphi(y), \varphi(x)], D(z)] \\
= & {[[D(x), \theta(y)], \varphi(z)]+(-1)^{d(D) d(x)} } \\
& \times[[\theta(x), D(y)], \theta(z)] \\
& +(-1)^{d(D)(d(x)+d(y))}[[\varphi(x), \varphi(y)], D(z)]
\end{aligned}
$$

then we have

$$
\begin{aligned}
& {[[D(x), \theta(y)], \theta(z)]} \\
& +\quad(-1)^{d(D) d(x)}[[\theta(x), D(y)], \varphi(z)] \\
& \quad=[[D(x), \theta(y)], \varphi(z)] \\
& \quad+(-1)^{d(D) d(x)}[[\theta(x), D(y)], \theta(z)] ;
\end{aligned}
$$

that is, $[[D(x), \theta(y)],(\varphi-\theta)(z)]=(-1)^{d(D) d(x)}[[\theta(x), D(y)]$, $(\varphi-\theta)(z)]$. It is routine to prove (ii).

Suppose, conversely, that $D$ is a Jordan $(\theta, \varphi)_{2}$ prederivation of $L$ satisfying (i) and (ii). Note that

$$
\begin{aligned}
& A^{\prime}(y, x, z) \\
&= {[[D(y), \theta(x)], \theta(z)] } \\
&+(-1)^{d(D) d(y)}[[\theta(y), D(x)], \varphi(z)] \\
&+(-1)^{d(D)(d(x)+d(y))}[[\varphi(y), \varphi(x)], D(z)] \\
&=-(-1)^{d(x) d(y)}[[D(x), \theta(y)], \varphi(z)]
\end{aligned}
$$

$$
\begin{aligned}
& -(-1)^{d(x) d(y)}(-1)^{d(D) d(x)}[[\theta(x), D(y)], \theta(z)] \\
& -(-1)^{d(x) d(y)}(-1)^{d(D)(d(x)+d(y))} \\
& \times[[\varphi(x), \varphi(y)], D(z)] \\
= & -(-1)^{d(x) d(y)}[[D(x), \theta(y)], \theta(z)] \\
& -(-1)^{d(x) d(y)}(-1)^{d(D) d(x)}[[\theta(x), D(y)], \varphi(z)] \\
& -(-1)^{d(x) d(y)}(-1)^{d(D)(d(x)+d(y))} \\
& \times[[\varphi(x), \varphi(y)], D(z)] \\
= & -(-1)^{d(x) d(y)} A^{\prime}(x, y, z) .
\end{aligned}
$$

In the same way, we can get equalities $(*)$ and $(* *)$. The rest of the proof is the same as the corresponding proof of Theorem 5.

A similar argument proves the following result.

Theorem 8. $D$ is a $(\theta, \varphi)_{3}$-prederivation of $L$ if and only if $D$ is a Jordan $(\theta, \varphi)_{3}$-prederivation of $L$ such that

(i) $[[D(x),(\theta-\varphi)(y)], \theta(z)]=(-1)^{d(D) d(x)}[[(\theta-$ $\varphi)(x), D(y)], \theta(z)]$,

(ii) $(-1)^{d(x) d(z)} A^{\prime \prime}(x, y, z)+(-1)^{d(x) d(y)} A^{\prime \prime}(y, z, x)+$ $(-1)^{d(y) d(z)} A^{\prime \prime}(z, x, y)=0$,

where $x, y, z \in L$ and $A^{\prime \prime}(x, y, z)=[[D(x), \theta(y)], \theta(z)]+$ $(-1)^{d(D) d(x)}[[\varphi(x), D(y)], \theta(z)]+(-1)^{d(D)(d(x)+d(y))}[[\varphi(x)$, $\varphi(y)], D(z)]$.

Remark 9. Corollary 6 can also be concluded from Theorem 7 or Theorem 8 since, for any $x, y, z \in L$, $A(x, y, z)=A^{\prime}(x, y, z)=A^{\prime \prime}(x, y, z)$ when $D$ is a Jordan $\theta$-prederivation.

Definition 10. Let $L$ be a Lie superalgebra.

(1) A generalized $(\theta, \varphi)_{1}$-prederivation with respect to a $(\theta, \varphi)_{1}$-prederivation $\delta_{1}$ is a homogeneous linear mapping $D_{1}: L \rightarrow L$ such that $d\left(D_{1}\right)=d\left(\delta_{1}\right)$ and

$$
\begin{aligned}
D_{1}([[x, y], z])= & {\left[\left[\delta_{1}(x), \theta(y)\right], \varphi(z)\right] } \\
& +(-1)^{d\left(D_{1}\right) d(x)}\left[\left[\theta(x), \delta_{1}(y)\right], \varphi(z)\right] \\
& +(-1)^{d\left(D_{1}\right)(d(x)+d(y))} \\
& \times\left[[\theta(x), \varphi(y)], D_{1}(z)\right], \quad \forall x, y, z \in L .
\end{aligned}
$$


(2) A generalized $(\theta, \varphi)_{2}$-prederivation with respect to a $(\theta, \varphi)_{2}$-prederivation $\delta_{2}$ is a homogeneous linear mapping $D_{2}: L \rightarrow L$ such that $d\left(D_{2}\right)=d\left(\delta_{2}\right)$ and

$$
\begin{aligned}
D_{2}([[x, y], z])= & {\left[\left[\delta_{2}(x), \theta(y)\right], \theta(z)\right] } \\
& +(-1)^{d\left(D_{2}\right) d(x)}\left[\left[\theta(x), \delta_{2}(y)\right], \varphi(z)\right] \\
& +(-1)^{d\left(D_{2}\right)(d(x)+d(y))} \\
& \times\left[[\varphi(x), \varphi(y)], D_{2}(z)\right], \quad \forall x, y, z \in L .
\end{aligned}
$$

(3) A generalized $(\theta, \varphi)_{3}$-prederivation with respect to a $(\theta, \varphi)_{3}$-prederivation $\delta_{3}$ is a homogeneous linear mapping $D_{3}: L \rightarrow L$ such that $d\left(D_{3}\right)=d\left(\delta_{3}\right)$ and

$$
\begin{aligned}
D_{3}([[x, y], z])= & {\left[\left[\delta_{3}(x), \theta(y)\right], \theta(z)\right] } \\
& +(-1)^{d\left(D_{3}\right) d(x)}\left[\left[\varphi(x), \delta_{3}(y)\right], \theta(z)\right] \\
& +(-1)^{d\left(D_{3}\right)(d(x)+d(y))} \\
& \times\left[[\varphi(x), \varphi(y)], D_{3}(z)\right], \quad \forall x, y, z \in L .
\end{aligned}
$$

In particular, $\forall i=1,2,3$, a generalized $(\theta, \varphi)_{i}$-prederivation $D$ is called a generalized $\theta$-prederivation with respect to a $\theta$ prederivation $\delta$ if $\theta=\varphi$. It is clear that $D$ is a generalized prederivation when $\theta=\varphi=1_{T}$ and $\delta$ is a prederivation.

Definition 11. Let $L$ be a Lie superalgebra.

(1) A generalized Jordan $(\theta, \varphi)_{1}$-prederivation with respect to a Jordan $(\theta, \varphi)_{1}$-prederivation $\delta_{1}$ is a homogeneous linear mapping $D_{1}: L \rightarrow L$ such that $d\left(D_{1}\right)=d\left(\delta_{1}\right)$ and

$$
\begin{aligned}
D_{1}([[x, y], x])= & {\left[\left[\delta_{1}(x), \theta(y)\right], \varphi(x)\right] } \\
& +(-1)^{d\left(D_{1}\right) d(x)}\left[\left[\theta(x), \delta_{1}(y)\right], \varphi(x)\right] \\
& +(-1)^{d\left(D_{1}\right)(d(x)+d(y))} \\
& \times\left[[\theta(x), \varphi(y)], D_{1}(x)\right], \quad \forall x, y \in L .
\end{aligned}
$$

(2) A generalized Jordan $(\theta, \varphi)_{2}$-prederivation with respect to a Jordan $(\theta, \varphi)_{2}$-prederivation $\delta_{2}$ is a homogeneous linear mapping $D_{2}: L \rightarrow L$ such that $d\left(D_{2}\right)=d\left(\delta_{2}\right)$ and

$$
\begin{aligned}
D_{2}([[x, y], x])= & {\left[\left[\delta_{2}(x), \theta(y)\right], \theta(x)\right] } \\
& +(-1)^{d\left(D_{2}\right) d(x)}\left[\left[\theta(x), \delta_{2}(y)\right], \varphi(x)\right] \\
& +(-1)^{d\left(D_{2}\right)(d(x)+d(y))} \\
& \times\left[[\varphi(x), \varphi(y)], D_{2}(x)\right], \quad \forall x, y \in L .
\end{aligned}
$$

(3) A generalized Jordan $(\theta, \varphi)_{3}$-prederivation with respect to a Jordan $(\theta, \varphi)_{3}$-prederivation $\delta_{3}$ is a homogeneous linear mapping $D_{3}: L \rightarrow L$ such that $d\left(D_{3}\right)=d\left(\delta_{3}\right)$ and

$$
\begin{aligned}
D_{3}([[x, y], x])= & {\left[\left[\delta_{3}(x), \theta(y)\right], \theta(x)\right] } \\
& +(-1)^{d\left(D_{3}\right) d(x)}\left[\left[\varphi(x), \delta_{3}(y)\right], \theta(x)\right] \\
& +(-1)^{d\left(D_{3}\right)(d(x)+d(y))} \\
& \times\left[[\varphi(x), \varphi(y)], D_{3}(x)\right], \quad \forall x, y \in L .
\end{aligned}
$$

In particular, $\forall i=1,2,3$, a generalized Jordan $(\theta, \varphi)_{i^{-}}$ prederivation $D$ is called a generalized Jordan $\theta$ prederivation with respect to a Jordan $\theta$-prederivation $\delta$ if $\theta=\varphi$. It is clear that $D$ is a generalized Jordan prederivation when $\theta=\varphi=1_{T}$ and $\delta$ is a Jordan prederivation.

Theorem 12. $D$ is a generalized $(\theta, \varphi)_{1}$-prederivation of $L$ with respect to $a(\theta, \varphi)_{1}$-prederivation $\delta$ if and only if $D$ is a generalized Jordan $(\theta, \varphi)_{1}$-prederivation of $L$ with respect to a $(\theta, \varphi)_{1}$-prederivation $\delta$ such that

(i) $[[\theta(x), \varphi(y)], D(z)]=[[\varphi(x), \theta(y)], D(z)]$,

(ii) $(-1)^{d(x) d(z)} B(x, y, z)+(-1)^{d(x) d(y)} B(y, z, x)+$ $(-1)^{d(y) d(z)} B(z, x, y)=0$,

where $x, y, z \in L$ and $B(x, y, z)=[[\delta(x), \theta(y)], \varphi(z)]+$ $(-1)^{d(D) d(x)}[[\theta(x), \delta(y)], \varphi(z)]+(-1)^{d(D)(d(x)+d(y))}[[\theta(x)$, $\varphi(y)], D(z)]$.

Proof. Suppose that $D$ is a generalized $(\theta, \varphi)_{1}$-prederivation of $L$ with respect to a $(\theta, \varphi)_{1}$-prederivation $\delta$. Clearly, $D$ is a generalized Jordan $(\theta, \varphi)_{1}$-prederivation of $L$ and $D([x, y, z])=-(-1)^{d(x) d(y)} D([y, x, z])$. (i) follows from the fact that

$$
\begin{aligned}
&-(-1)^{d(x) d(y)} D([[y, x], z]) \\
&=-(-1)^{d(x) d(y)}[[\delta(y), \theta(x)], \varphi(z)] \\
& \quad-(-1)^{d(x) d(y)}(-1)^{d(D) d(y)}[[\theta(y), \delta(x)], \varphi(z)] \\
& \quad-(-1)^{d(x) d(y)}(-1)^{d(D)(d(x)+d(y))} \\
& \quad \times[[\theta(y), \varphi(x)], D(z)] \\
&= {[[\delta(x), \theta(y)], \varphi(z)]+(-1)^{d(D) d(x)} } \\
& \times[[\theta(x), \delta(y)], \varphi(z)] \\
&+(-1)^{d(D)(d(x)+d(y))}[[\varphi(x), \theta(y)], D(z)] .
\end{aligned}
$$


Since $D$ is a generalized $(\theta, \varphi)_{1}$-prederivation of $L$ with respect to $\delta$, we have $D([[x, y], z])=B(x, y, z)$; hence

$$
\begin{aligned}
(-1)^{d(x) d(z)} B(x, y, z)+(-1)^{d(x) d(y)} B(y, z, x) \\
\quad+(-1)^{d(y) d(z)} B(z, x, y) \\
=(-1)^{d(x) d(z)} D([[x, y], z]) \\
\quad+(-1)^{d(x) d(y)} D([[y, z], x]) \\
\quad+(-1)^{d(y) d(z)} D([[z, x], y]) \\
=D\left((-1)^{d(x) d(z)}[[x, y], z]+(-1)^{d(x) d(y)}[[y, z], x]\right. \\
\left.\quad+(-1)^{d(y) d(z)}[[z, x], y]\right)=0 .
\end{aligned}
$$

Conversely, if $D$ is a generalized Jordan $(\theta, \varphi)_{1}$-prederivation of $L$ with respect to a $(\theta, \varphi)_{1}$-prederivation $\delta$ satisfying (i) and (ii), then refer to the proof of Theorem 5; it suffices to prove $B(x, y, z)=-(-1)^{d(x) d(y)} B(y, x, z)$. In fact,

$$
\begin{aligned}
& B(y, x, z) \\
&= {[\delta(y), \theta(x)], \varphi(z)] } \\
&+(-1)^{d(D) d(y)}[[\theta(y), \delta(x)], \varphi(z)] \\
&+(-1)^{d(D)(d(x)+d(y))}[[\theta(y), \varphi(x)], D(z)] \\
&=-(-1)^{d(x) d(y)}[[\delta(x), \theta(y)], \varphi(z)] \\
&-(-1)^{d(x) d(y)}(-1)^{d(D) d(x)}[[\theta(x), \delta(y)], \varphi(z)] \\
&-(-1)^{d(x) d(y)}(-1)^{d(D)(d(x)+d(y))} \\
& \times[[\varphi(x), \theta(y)], D(z)] \\
&=-(-1)^{d(x) d(y)}[[\delta(x), \theta(y)], \varphi(z)] \\
&-(-1)^{d(x) d(y)}(-1)^{d(D) d(x)} \\
& \times[[\theta(x), \delta(y)], \varphi(z)] \\
&-(-1)^{d(x) d(y)}(-1)^{d(D)(d(x)+d(y))} \\
& \times[[\theta(x), \varphi(y)], D(z)] \\
&=-(-1)^{d(x) d(y)} B(x, y, z) . \\
&
\end{aligned}
$$

This completes the proof.

Corollary 13. $D$ is a generalized $\theta$-prederivation of $L$ with respect to a $\theta$-prederivation $\delta$ if and only if $D$ is a generalized
Jordan $\theta$-prederivation of $L$ with respect to a Jordan $\theta$ prederivation $\delta$ such that

$$
\begin{aligned}
& (-1)^{d(z)(d(D)+d(x))}[[\theta(x), \theta(y)],(D-\delta)(z)] \\
& \quad+(-1)^{d(x)(d(D)+d(y))}[[\theta(y), \theta(z)],(D-\delta)(x)] \\
& \quad+(-1)^{d(y)(d(D)+d(z))}[[\theta(z), \theta(x)],(D-\delta)(y)]=0 .
\end{aligned}
$$

Proof. If $D$ is a generalized Jordan $\theta$-prederivation of $L$, then (i) follows immediately. (ii) holds because

$$
\begin{aligned}
& (-1)^{d(x) d(z)} B(x, y, z)+(-1)^{d(x) d(y)} B(y, z, x) \\
& +(-1)^{d(y) d(z)} B(z, x, y) \\
& =(-1)^{d(x) d(z)}[[\delta(x), \theta(y)], \theta(z)] \\
& +(-1)^{d(x) d(z)}(-1)^{d(D) d(x)}[[\theta(x), \delta(y)], \theta(z)] \\
& +(-1)^{d(x) d(z)}(-1)^{d(D)(d(x)+d(y))}[[\theta(x), \theta(y)], D(z)] \\
& +(-1)^{d(x) d(y)}[[\delta(y), \theta(z)], \theta(x)] \\
& +(-1)^{d(x) d(y)}(-1)^{d(D) d(y)}[[\theta(y), \delta(z)], \theta(x)] \\
& +(-1)^{d(x) d(y)}(-1)^{d(D)(d(y)+d(z))}[[\theta(y), \theta(z)], D(x)] \\
& +(-1)^{d(y) d(z)}[[\delta(z), \theta(x)], \theta(y)] \\
& +(-1)^{d(y) d(z)}(-1)^{d(D) d(z)}[[\theta(z), \delta(x)], \theta(y)] \\
& +(-1)^{d(y) d(z)}(-1)^{d(D)(d(x)+d(z))}[[\theta(z), \theta(x)], D(y)] \\
& =(-1)^{d(x) d(z)}[[\delta(x), \theta(y)], \theta(z)] \\
& +(-1)^{d(x) d(z)}(-1)^{d(D) d(x)}[[\theta(x), \delta(y)], \theta(z)] \\
& +(-1)^{d(x) d(z)}(-1)^{d(D)(d(x)+d(y))}[[\theta(x), \theta(y)], \delta(z)] \\
& +(-1)^{d(x) d(y)}[[\delta(y), \theta(z)], \theta(x)] \\
& +(-1)^{d(x) d(y)}(-1)^{d(D) d(y)}[[\theta(y), \delta(z)], \theta(x)] \\
& +(-1)^{d(x) d(y)}(-1)^{d(D)(d(y)+d(z))}[[\theta(y), \theta(z)], \delta(x)] \\
& +(-1)^{d(y) d(z)}[[\delta(z), \theta(x)], \theta(y)] \\
& +(-1)^{d(y) d(z)}(-1)^{d(D) d(z)}[[\theta(z), \delta(x)], \theta(y)] \\
& +(-1)^{d(y) d(z)}(-1)^{d(D)(d(x)+d(z))}[[\theta(z), \theta(x)], \delta(y)] \\
& =(-1)^{d(D) d(z)}
\end{aligned}
$$




$$
\begin{aligned}
& \times\left((-1)^{d(z)(d(D)+d(x))}[[\delta(x), \theta(y)], \theta(z)]\right. \\
& +(-1)^{d(y)(d(D)+d(x))}[[\theta(y), \theta(z)], \delta(x)] \\
& \left.\quad+(-1)^{d(y) d(z)}[[\theta(z), \delta(x)], \theta(y)]\right) \\
& +(-1)^{d(D) d(x)} \\
& \times\left((-1)^{d(x)(d(D)+d(y))}[[\delta(y), \theta(z)], \theta(x)]\right. \\
& \quad+(-1)^{d(z)(d(D)+d(y))}[[\theta(z), \theta(x)], \delta(y)] \\
& \left.\quad+(-1)^{d(x) d(z)}[[\theta(x), \delta(y)], \theta(z)]\right) \\
& +(-1)^{d(D) d(y)} \\
& \times\left((-1)^{d(y)(d(D)+d(z))}[[\delta(z), \theta(x)], \theta(y)]\right. \\
& \quad+(-1)^{d(x)(d(D)+d(z))}[[\theta(x), \theta(y)], \delta(z)] \\
& \left.\quad+(-1)^{d(x) d(y)}[[\theta(y), \delta(z)], \theta(x)]\right)=0 .
\end{aligned}
$$

Note that $\delta$ is a $\theta$-prederivation by Corollary 6 . Therefore, $D$ is a generalized $\theta$-prederivation of $L$ with respect to a $\theta$ prederivation $\delta$ by Theorem 12 .

As before, one can prove the following theorems.

Theorem 14. $D$ is a generalized $(\theta, \varphi)_{2}$-prederivation of $L$ with respect to a $(\theta, \varphi)_{2}$-prederivation $\delta$ if and only if $D$ is a generalized Jordan $(\theta, \varphi)_{2}$-prederivation of $L$ with respect to a $(\theta, \varphi)_{2}$-prederivation $\delta$ such that

(i) $[[\delta(x), \theta(y)],(\varphi-\theta)(z)]=(-1)^{d(\delta) d(x)}[[\theta(x), \delta(y)]$, $(\varphi-\theta)(z)]$

(ii) $(-1)^{d(x) d(z)} B^{\prime}(x, y, z)+(-1)^{d(x) d(y)} B^{\prime}(y, z, x)+$ $(-1)^{d(y) d(z)} B^{\prime}(z, x, y)=0$,

where $x, y, z \in L$ and $B^{\prime}(x, y, z)=[[\delta(x), \theta(y)], \theta(z)]+$ $(-1)^{d(D) d(x)}[[\theta(x), \delta(y)], \varphi(z)]+(-1)^{d(D)(d(x)+d(y))}[[\varphi(x)$, $\varphi(y)], D(z)]$.

Theorem 15. $D$ is a generalized $(\theta, \varphi)_{3}$-prederivation of $L$ with respect to a $(\theta, \varphi)_{3}$-prederivation $\delta$ if and only if $D$ is a generalized Jordan $(\theta, \varphi)_{3}$-prederivation of $L$ with respect to a $(\theta, \varphi)_{3}$-prederivation $\delta$ such that

(i) $[[\delta(x),(\theta-\varphi)(y)], \theta(z)]=(-1)^{d(\delta) d(x)}[[(\theta-\varphi)(x)$, $\delta(y)], \theta(z)]$

(ii) $(-1)^{d(x) d(z)} B^{\prime \prime}(x, y, z)+(-1)^{d(x) d(y)} B^{\prime \prime}(y, z, x)+$ $(-1)^{d(y) d(z)} B^{\prime \prime}(z, x, y)=0$,

where $x, y, z \in L$ and $B^{\prime \prime}(x, y, z)=[[\delta(x), \theta(y)], \theta(z)]+$ $(-1)^{d(\delta) d(x)}[[\varphi(x), \delta(y)], \theta(z)]+(-1)^{d(D)(d(x)+d(y))}[[\varphi(x)$, $\varphi(y)], D(z)]$.
Remark 16. Corollary 13 can also be concluded from Theorem 14 or Theorem 15 since, for any $x, y, z \in L$, $B(x, y, z)=B^{\prime}(x, y, z)=B^{\prime \prime}(x, y, z)$ when $D$ is a generalized Jordan $\theta$-prederivation.

\section{Conflict of Interests}

The authors declare that there is no conflict of interests regarding the publication of this paper.

\section{Acknowledgments}

The paper is supported by NNSF of China (no. 11471090 and no. 11171055), Natural Science Foundation of Jilin Province (no. 201115006), Scientific Research Foundation for Returned Scholars, Ministry of Education of China, and the Fundamental Research Funds for the Central Universities (no. 12SSXT139).

\section{References}

[1] G. F. Leger and E. M. Luks, "Generalized derivations of lie algebras," Journal of Algebra, vol. 228, no. 1, pp. 165-203, 2000.

[2] C.-K. Liu and W.-K. Shiue, "Generalized Jordan triple $(\theta, \varphi)$ derivations on semiprime rings," Taiwanese Journal of Mathematics, vol. 11, no. 5, pp. 1397-1406, 2007.

[3] A. Najati, "Jordan $\theta$-derivations on Lie triple systems," Bulletin of the Korean Mathematical Society, vol. 46, no. 3, pp. 435-437, 2009.

[4] Y. Benoist, "Semisimple part of the algebra of derivations of a nilpotent Lie algebra," Comptes Rendus de l'Académie des Sciences. Series I. Mathematics, vol. 307, no. 18, pp. 901-904, 1988.

[5] I. N. Herstein, Topics in Ring Theory, The University of Chicago Press, Chicago, Ill, USA, 1969.

[6] M. Brešar and J. Vukman, "Jordan $(\Theta, \varphi)$-derivations," Glasnik Matematicki, vol. 26, no. 1-2, pp. 13-17, 1991.

[7] M. Ashraf, A. Ali, and S. Ali, "On Lie ideals and generalized ( $\theta$, $\varphi)$-derivations in prime rings," Communications in Algebra, vol. 32, no. 8, pp. 2977-2985, 2004.

[8] L. Chen, Y. Ma, and L. Ni, "Generalized derivations of lie color algebras," Results in Mathematics, vol. 63, no. 3-4, pp. 923-936, 2013.

[9] A. Najati, "Generalized derivations on Lie triple systems," Results in Mathematics, vol. 54, no. 1-2, pp. 143-147, 2009.

[10] A. Najati, "On generalized Jordan derivations of Lie triple systems," Czechoslovak Mathematical Journal, vol. 60(135), no. 2, pp. 541-547, 2010.

[11] D. Müller, "Isometries of bi-invariant pseudo-Riemannian metrics on Lie groups," Geometriae Dedicata, vol. 29, no. 1, pp. 6596, 1989.

[12] I. Bajo, "Lie algebras admitting non-singular prederivations," Indagationes Mathematicae: New Series, vol. 8, no. 4, pp. 433437, 1997.

[13] W. A. Moens, "A characterisation of nilpotent Lie algebras by invertible LEIbniz-derivations," Communications in Algebra, vol. 41, no. 7, pp. 2427-2440, 2013.

[14] D. Burde, "Affine cohomology classes for filiform Lie algebras," Contemporary Mathematics, vol. 262, pp. 159-170, 2000. 
[15] D. Burde, "Lie algebra prederivations and strongly nilpotent Lie algebras," Communications in Algebra, vol. 30, no. 7, pp. 31573175, 2002.

[16] D. Burde and W. A. Moens, "Periodic derivations and prederivations of Lie algebras," Journal of Algebra, vol. 357, pp. 208-221, 2012.

[17] P. Ji and L. Wang, "Lie triple derivations of TUHF algebras," Linear Algebra and Its Applications, vol. 403, no. 1-3, pp. 399408, 2005.

[18] C. R. Miers, "Lie triple derivations of von Neumann algebras," Proceedings of the American Mathematical Society, vol. 71, no. 1, pp. 57-61, 1978.

[19] H.-T. Wang and Q.-G. Li, "Lie triple derivation of the Lie algebra of strictly upper triangular matrix over a commutative ring," Linear Algebra and Its Applications, vol. 430, no. 1, pp. 66-77, 2009.

[20] J. Zhang, B. Wu, and H. Cao, "Lie triple derivations of nest algebras," Linear Algebra and Its Applications, vol. 416, no. 2-3, pp. 559-567, 2006.

[21] M. Brešar, "Jordan mappings of semiprime rings," Journal of Algebra, vol. 127, no. 1, pp. 218-228, 1989. 


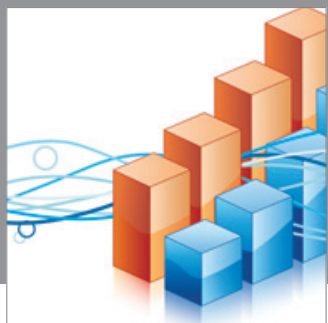

Advances in

Operations Research

mansans

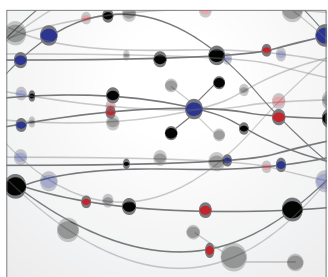

The Scientific World Journal
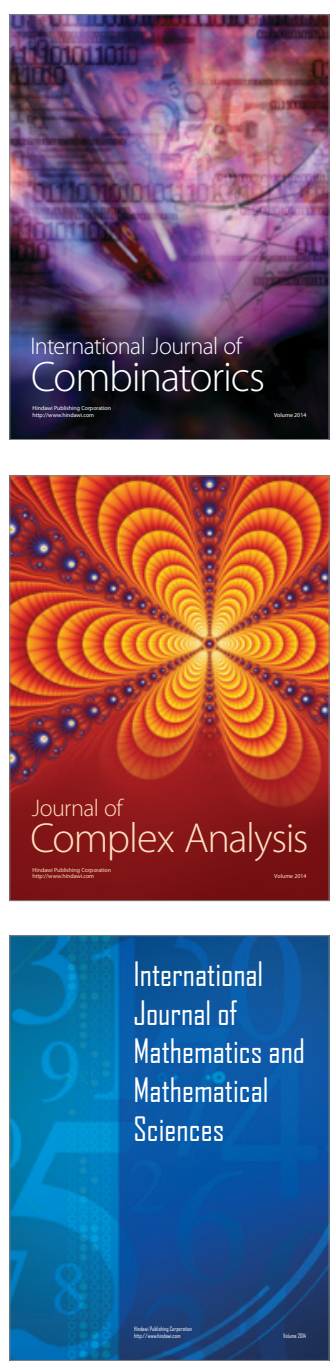
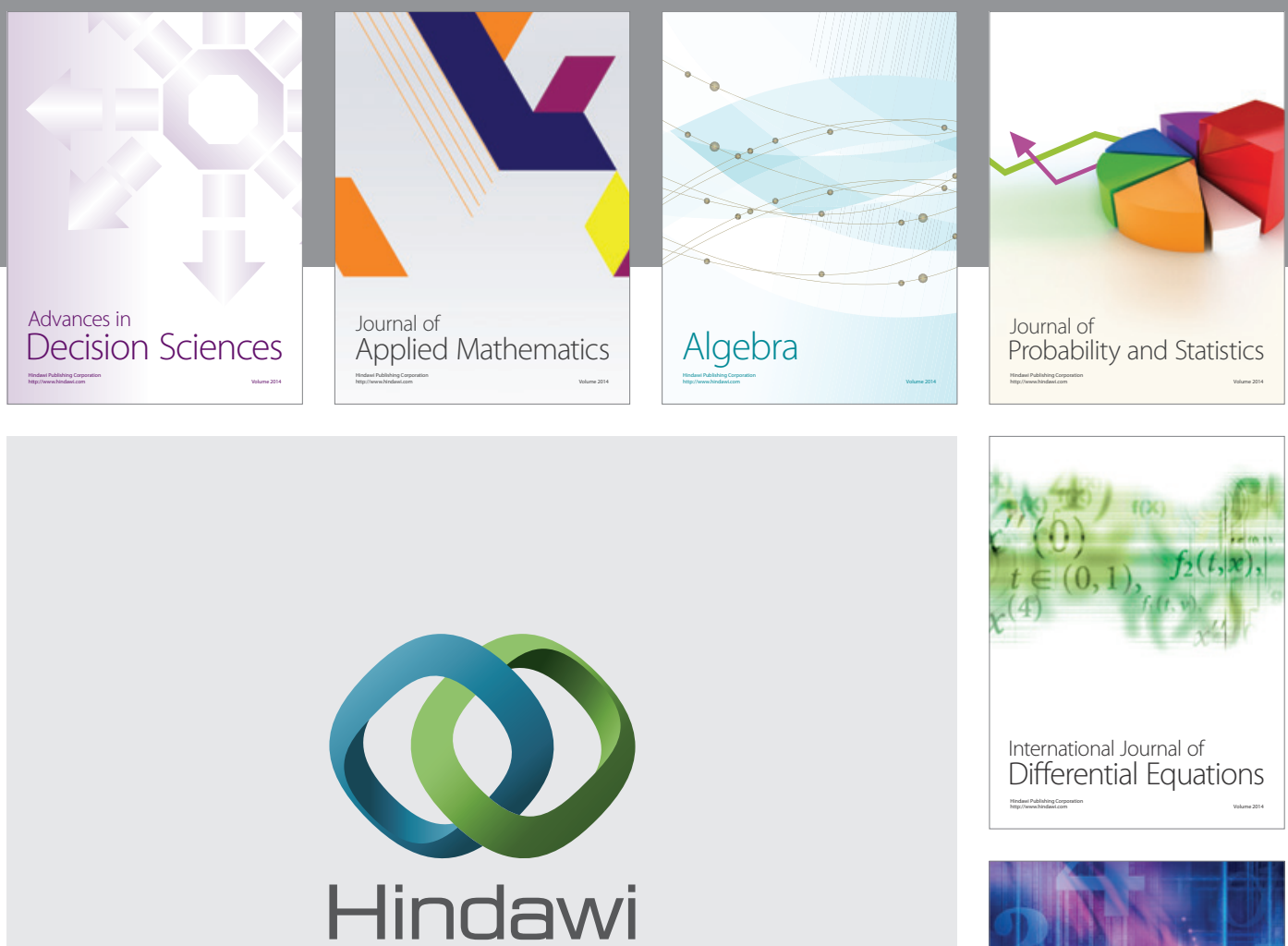

Submit your manuscripts at http://www.hindawi.com
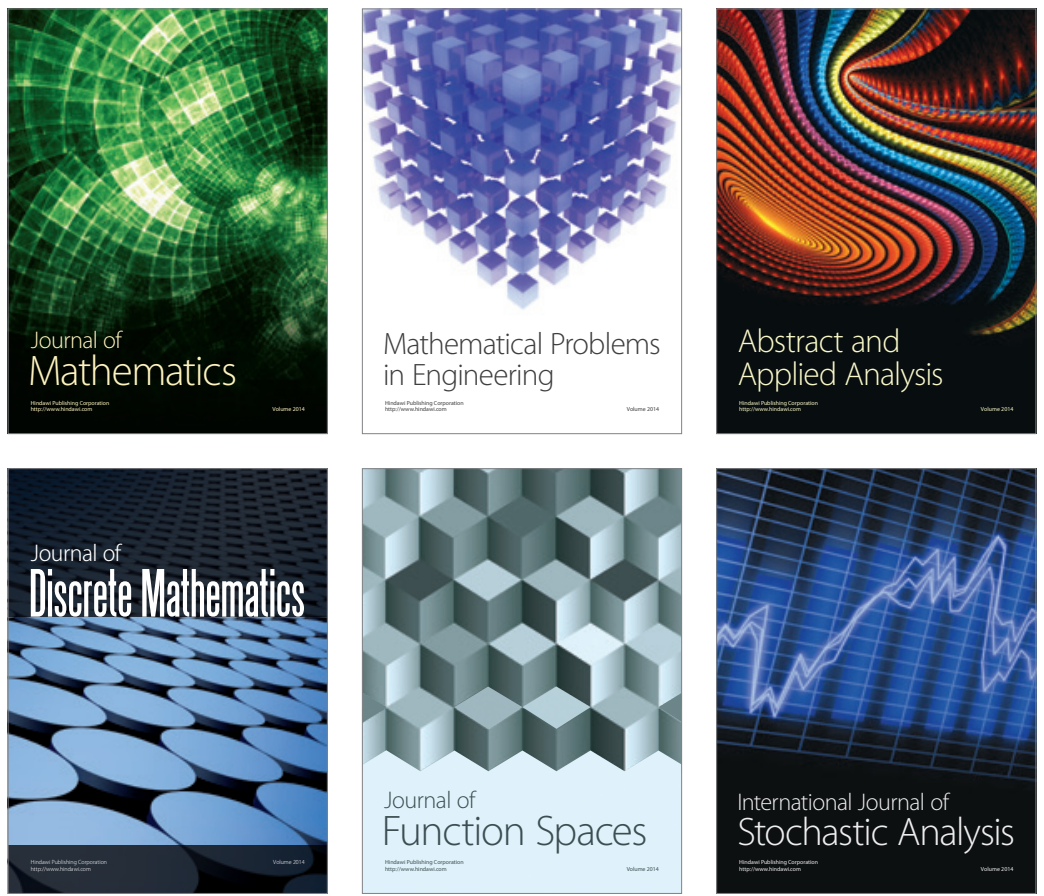

Journal of

Function Spaces

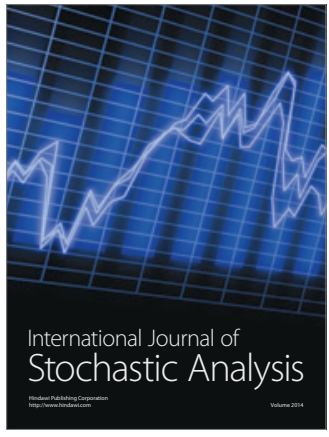

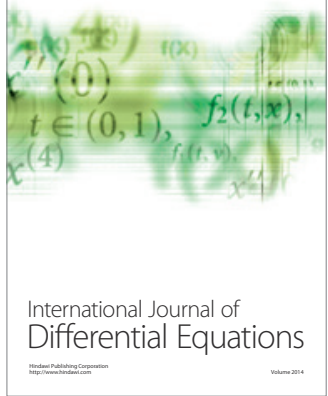
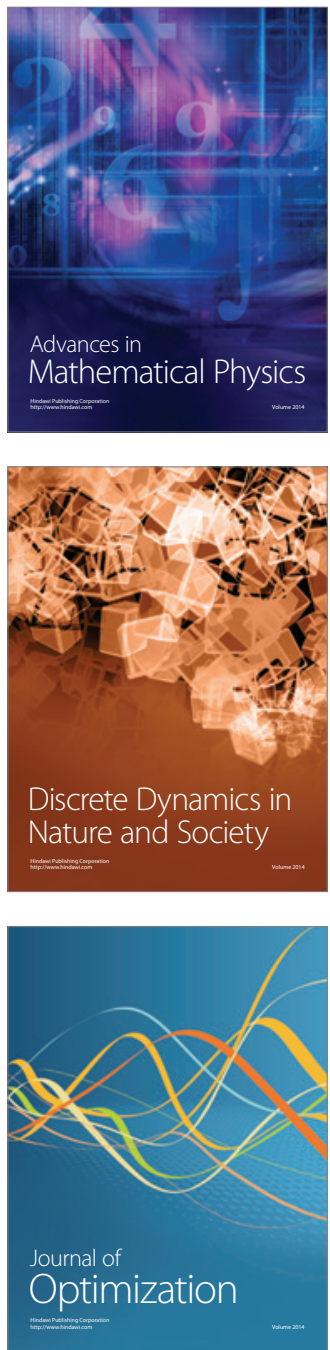